\title{
The impact of high functional connectivity network hub resection on language task performance in adult low- and high-grade glioma
}

\author{
*Anthony T. Lee, MD, PhD, ${ }^{1}$ Claire Faltermeier, MD, PhD, ${ }^{1}$ Ramin A. Morshed, MD, ${ }^{1}$ \\ Jacob S. Young, MD, ${ }^{1}$ Sofia Kakaizada, BS, ${ }^{1}$ Claudia Valdivia, BA, ${ }^{1}$ Anne M. Findlay, MA, ${ }^{2}$ \\ Phiroz E. Tarapore, MD, ${ }^{1}$ Srikantan S. Nagarajan, PhD, ${ }^{2}$ Shawn L. Hervey-Jumper, MD, ${ }^{1}$ and \\ Mitchel S. Berger, MD' \\ 1Department of Neurological Surgery and 'Biomagnetic Imaging Laboratory, Department of Radiology and Biomedical Imaging, \\ University of California, San Francisco, California
}

\begin{abstract}
OBJECTIVE Gliomas are intrinsic brain tumors with the hallmark of diffuse white matter infiltration, resulting in shortand long-range network dysfunction. Preoperative magnetoencephalography (MEG) can assist in maximizing the extent of resection while minimizing morbidity. While MEG has been validated in motor mapping, its role in speech mapping remains less well studied. The authors assessed how the resection of intraoperative electrical stimulation (IES)-negative, high functional connectivity (HFC) network sites, as identified by MEG, impacts language performance.

METHODS Resting-state, whole-brain MEG recordings were obtained from 26 patients who underwent perioperative language evaluation and glioma resection that was guided by awake language and IES mapping. The functional connectivity of an individual voxel was determined by the imaginary coherence between the index voxel and the rest of the brain, referenced to its contralesional pair. The percentage of resected HFC voxels was correlated with postoperative language outcomes in tasks of increasing complexity: text reading, 4-syllable repetition, picture naming, syntax (SYN), and auditory stimulus naming (AN).
\end{abstract}

RESULTS Overall, $70 \%$ of patients (14/20) in whom any HFC tissue was resected developed an early postoperative language deficit (mean 2.3 days, range 1-8 days), compared to $33 \%$ of patients (2/6) in whom no HFC tissue was resected $(p=0.16)$. When bifurcated by the amount of HFC tissue that was resected, $100 \%$ of patients $(3 / 3)$ with an HFC resection $>25 \%$ displayed deficits in AN, compared to $30 \%$ of patients $(6 / 20)$ with an HFC resection $<25 \%(p=0.04)$. Furthermore, there was a linear correlation between the severity of AN and SYN decline with percentage of HFC sites resected $(p=0.02$ and $p=0.04$, respectively). By 2.2 months postoperatively (range 1-6 months), the correlation between HFC resection and both AN and SYN decline had resolved ( $p=0.94$ and $p=1.00$, respectively) in all patients $(9 / 9)$ except two who experienced early postoperative tumor progression or stroke involving inferior frontooccipital fasciculus.

CONCLUSIONS Imaginary coherence measures of functional connectivity using MEG are able to identify HFC network sites within and around low- and high-grade gliomas. Removal of IES-negative HFC sites results in early transient postoperative decline in AN and SYN, which resolved by 3 months in all patients without stroke or early tumor progression. Measures of functional connectivity may therefore be a useful means of counseling patients about postoperative risk and assist with preoperative surgical planning.

https://thejns.org/doi/abs/10.3171/2020.1.JNS192267

KEYWORDS glioblastoma; low-grade glioma; high-grade glioma; language; speech; functional connectivity; magnetoencephalography; oncology

ABBREVIATIONS ADP = afterdischarge potential; $\mathrm{AN}=$ auditory stimulus naming; $\mathrm{DWI}=$ diffusion weighted imaging; $\mathrm{HFC}=$ high functional connectivity; IES = intraoperative electrical stimulation; LFC = low functional connectivity; MEG = magnetoencephalography; $\mathrm{PN}$ = picture naming; $\mathrm{QAB}=$ quick aphasia battery; $\mathrm{SYN}=$ syntax; TR = single-word text reading; UCSF = University of California, San Francisco; 4-SYL = 4-syllable repetition.

SUBMITTED August 19, 2019. ACCEPTED January 13, 2020.

INCLUDE WHEN CITING Published online April 3, 2020; DOI: 10.3171/2020.1.JNS192267.

${ }^{*}$ A.T.L. and C.F. contributed equally to this work, and S.L.H.J. and M.S.B. contributed equally to this work. 
$\mathrm{M}$ AXIMIZING the extent of tumor resection while minimizing neurological morbidity is a central tenant of neurological surgery. ${ }^{14,28,30,31,34}$ Functionally significant regions of brain are unique across patients due to distortion of cortical topography from mass effect, as well as the functional reorganization caused by neuroplasticity in response to tumor invasion. ${ }^{10,24,38}$ Direct intraoperative electrical stimulation (IES) remains the gold standard for the mapping of lesional and perilesional tissue in functional brain regions. Advances in functional neuroimaging such as PET, functional MRI, and magnetoencephalography (MEG) may be utilized to generate functional maps to aid in surgical planning and patient counseling. Combined with noninvasive neurostimulation modalities such as transcranial magnetic stimulation, ${ }^{37}$ neurosurgeons have several options to help them understand the risks associated with surgical strategies. Patients with baseline aphasia who might not otherwise be able to participate in awake IES language mapping may benefit from these alternative mapping modalities.

MEG captures direct measures of neuronal activity with high spatiotemporal resolution by uncovering changes in magnetic fields generated from postsynaptic currents. ${ }^{13}$ Functional connectivity is a measure of the neuronal synchrony between the voxel of interest and other brain areas. Regions with high functional connectivity (HFC) share similar functional interactions, while regions with low functional connectivity (LFC) are relatively dissimilar in their neuronal activity. Disruptions in functional connectivity occur in patients with intrinsic brain tumors and correlate with global neurocognitive dysfunction. ${ }^{3,4,6,7}$ Prior work from our group has shown MEG imaging of resting-state functional connectivity to be a useful noninvasive means of evaluating the function of lesional and perilesional brain tissue for surgical planning in cases of tumor resection. ${ }^{12}$ For example, functional maps of LFC sites were found to have a $100 \%$ negative predictive value for the presence of eloquent cortex during IES, ${ }^{20}$ motor evoked MEG signals mapped onto IES motor sites to within a $1-\mathrm{cm}$ margin, ${ }^{37}$ and patients with resection of tumors with LFC tissue had a 0\% rate of new neurological deficit at the 6-month follow-up. ${ }^{36}$

Although the role of MEG has been well validated for preoperative motor and somatosensory mapping, ${ }^{19,21,33,45}$ less is known about its correlation to intraoperative speech mapping. Specifically, how HFC (vs LFC) network hubs contribute to expressive and receptive language processing remains unclear. In this study, we examined postoperative language outcomes using tasks of varying complexity following removal of intra- and peritumoral HFC network hubs during tumor resection. We test the hypothesis that removal of IESnegative HFC network sites will correlate with declined language task performance. Patients underwent extensive perioperative language assessment and received tailored tumor resection guided by IES awake language mapping according to standard-of-care clinical protocol.

\section{Methods}

\section{Patients and Outcome Measurements}

We reviewed a prospective registry of adult glioma patients that included 26 patients with left-sided focal low- and high-grade gliomas, detailed language assessments, and baseline MEG findings. All procedures were performed at the University of California, San Francisco (UCSF), by two surgeons (M.S.B. and S.L.H.J.) between September 2017 and December 2018. Preoperative MEG was performed at the UCSF Biomagnetic Imaging Laboratory. All patients gave written informed consent and the Institutional Review Board at UCSF approved this study.

\section{Imaging Acquisition and MEG Data Analysis}

High-resolution MRI was performed using a 3-T unit to provide anatomical detail for surgical planning and intraoperative neuronavigation. The protocol included the following sequences: 1) a T1-weighted, 3D, spoiled gradient-recalled acquisition in steady-state sequence, with TR 6-9 msec, TE $2-3 \mathrm{msec}$, and flip angle $12^{\circ}-15^{\circ}$; and 2) a T2-weighted, $3 \mathrm{D}$, fast spin echo sequence, with $T R$ 2000-3800 msec and TE 87-159 msec. Both sequences had a slice thickness between 1 and $1.5 \mathrm{~mm}$, an acquisition matrix from $256 \times 256$ to $288 \times 288$, contained between 114 and 428 slices, and included all fiducial points and markers.

Details of the MEG recording and analysis can be found in prior studies. ${ }^{20,27,36,37}$ In summary, patients were lying awake with their eyes closed in a magnetically shielded room while a 275-channel whole-head CTF Omega 2000 system (CTF Systems, Inc.) captured their continuous resting-state MEG using a sampling rate of $1200 \mathrm{~Hz}$. The locations of the MEG coils were triangulated at the beginning and end of the recording run and later coregistered to a structural MR image to generate the head shape. An artifact-free 1-minute epoch was selected for further analysis if the patient's head movement did not exceed $0.5 \mathrm{~cm}$.

This artifact-free, 1-minute epoch was then analyzed using the NUTMEG software suite (UCSF Biomagnetic Imaging Laboratory) to reconstruct whole-brain oscillatory activity from MEG sensors so as to construct functional connectivity (imaginary coherence) metrics., ${ }^{9,17,44}$ Spatially normalized structural MR images were used to overlay a volume-of-interest projection (grid size $=8 \mathrm{~mm}$; approximately 3000 voxels/subject) such that each voxel contained the entire time series of activity for that location derived by all the MEG sensor recordings. The time series within each voxel was then bandpass filtered for the alpha band $(1-20 \mathrm{~Hz})$ and reconstructed in source space using a minimum-variance adaptive spatial filtering technique.,94 The alpha band was selected because it was the most consistently identified peak in the power spectra from this sampling window in our patient series. The imaginary coherence technique was used to estimate functional connectivity because it has been previously been shown to reduce overestimation biases in MEG data. ${ }^{12,22}$

\section{Functional Connectivity Maps}

The functional connectivity of an individual voxel was derived by the mean imaginary coherence between the index voxel and the rest of the brain, referenced to its contralesional pair. ${ }^{36}$ Comparisons to contralesional vox- 
els used a 2-tailed t-test to test the null hypothesis that the Z-transformed connectivity imaginary coherence between the index voxel and nontumor voxel is equal to the mean of the Z-transformed connectivity between all contralateral voxels and the same set of voxels. The resultant functional connectivity values were separated into tertiles: upper tertile (high connectivity), middle tertile (neutral connectivity), and lower tertile (low connectivity). Functional connectivity maps were created by projecting connectivity data onto each individual patient's preoperative structural MR images. Preoperative functional connectivity maps were overlaid with postoperative MR images to determine whether HFC hubs had been resected. Side-byside comparisons of preoperative and postoperative MR images were used to determine the number of HFC voxels that had been resected, and this was independently validated by two researchers.

\section{IES Mapping}

The intraoperative awake mapping techniques used in this study have been described previously. $15,18,23,29,30,35$ In brief, frameless navigational guidance based on preoperative MR images was used to tailor a craniotomy for exposure of the tumor with a surrounding $2-\mathrm{cm}$ border. The targeted area for resection involved hyperintense signals on T2-weighted FLAIR images for low-grade glioma and T1-weighted post-Gd contrast-enhancing regions for highgrade glioma. A 1-mm bipolar electrode separated by 5 $\mathrm{mm}$ was used to perform IES of the cerebral cortex and subcortical white matter with electrocorticography for detection of afterdischarge potentials (ADPs) and subclinical seizure activity. Termination of stimulation-induced seizures was first attempted with cold Ringer's solution, ${ }^{32}$ followed by intravenous propofol $(1 \mathrm{mg} / \mathrm{kg})$ if seizures remained refractory to cold irrigation. Stimulation intensity started at $2 \mathrm{~mA}$ and was increased to a maximum of $6 \mathrm{~mA}$, or $1 \mathrm{~mA}$ less than the intensity of the ADPs if ADPs were detected. Intraoperative language testing was performed as previously described.$^{30}$ Intraoperative language testing included picture naming (PN) of visual stimuli, single-word text reading (TR), and short-phrase sentence completion. Positive language sites were defined by stimulation-induced anomia, alexia, or speech arrest during 2 of 3 stimulation trials. The decision regarding resection of tumor and peritumor cortical and subcortical sites was made purely based on IES mapping in-line with established clinical protocol (not MEG functional connectivity measures). ${ }^{15}$ Patients received a standardized decadron regimen: Decadron $4 \mathrm{mg}$ every 8 hours, with a 14-day taper off (if any baseline language task dysfunction were present) or $2 \mathrm{mg}$ twice a day, with a 4-day taper off.

\section{Language Evaluation and Functional Outcome Testing}

One day prior to tumor resection, patients underwent baseline language evaluation consisting of 6 tests. The quick aphasia battery (QAB) was designed as an efficient, reliable, multidimensional assessment of language function that surveys 7 language domains (word comprehension, sentence comprehension, word finding, grammatical construction, speech motor programming, repetition,
Text Reading

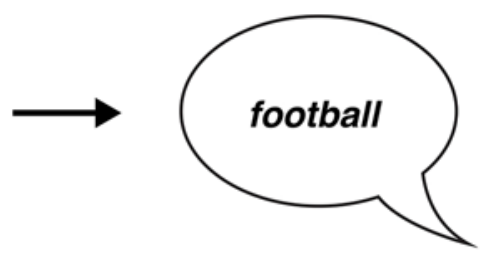

\section{4-Syllable Repetition}
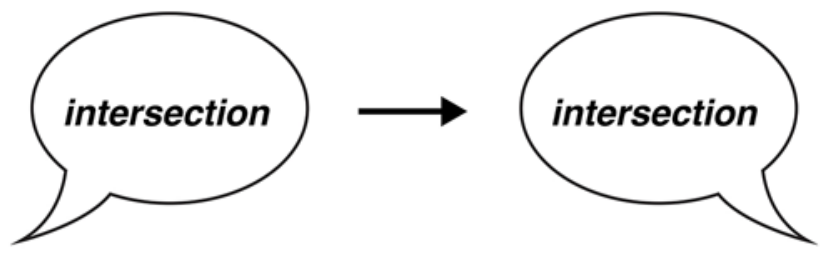

\section{Picture Naming}
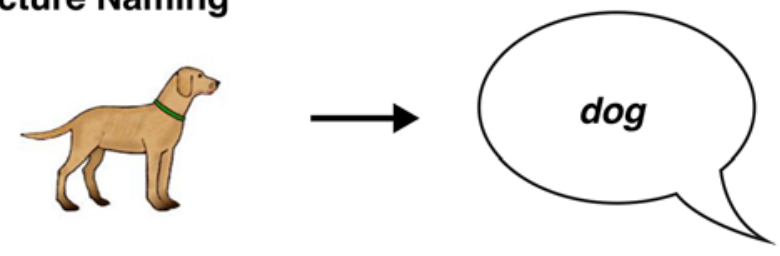

\section{Syntax}
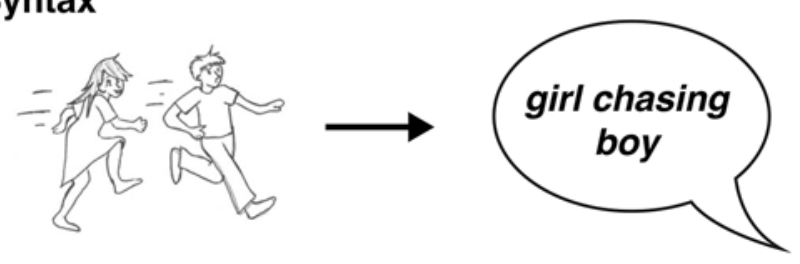

\section{Auditory Naming}
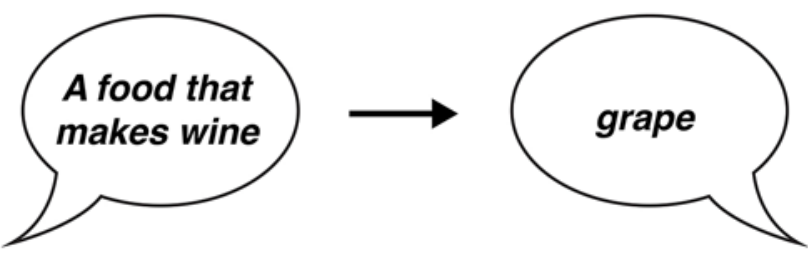

FIG. 1. Examples of the tasks used to assess postoperative language outcomes. Each task assesses overlapping, but distinct, cognitive domains used in language processing. Figure is available in color online only.

and reading) for an overall composite QAB score. ${ }^{42}$ To increase the sensitivity of detecting dysfunction in specific language domains, the multidimensional $\mathrm{QAB}$ was supplemented by TR (27 trials), 4-syllable repetition (4SYL, 87 trials), PN (48 trials), syntax (SYN, 28 trials), and auditory stimulus naming (AN, 32 trials) tests (Fig. 1). In TR, patients were asked to read aloud texts of single monosyllabic and disyllabic words, and in the 4-SYL test, patients were instructed to repeat 4-syllable words. In PN, patients named simple line-drawn objects. In SYN tests, patients examined drawings of two characters and were 
TABLE 1. Demographic and clinical characteristics

\begin{tabular}{|c|c|c|c|}
\hline \multirow[b]{2}{*}{ Characteristic } & \multicolumn{3}{|c|}{ Postoperative Language Decline } \\
\hline & $\begin{array}{c}\text { No } \\
(n=10)\end{array}$ & $\begin{array}{c}\text { Yes } \\
(n=16)\end{array}$ & $\begin{array}{c}p \\
\text { Value }\end{array}$ \\
\hline Sex, no. $(\%)$ & & & 0.22 \\
\hline Female & $2(20)$ & $8(80)$ & \\
\hline Male & $8(50)$ & $8(50)$ & \\
\hline Age (range), yrs & $48.4(21-69)$ & $52.7(20-80)$ & 0.77 \\
\hline Language evaluation, POD (range) & $2.30(1-8)$ & $2.31(1-8)$ & 0.49 \\
\hline Handedness, no. (\%) & & & 0.04 \\
\hline Right & $7(30)$ & $16(70)$ & \\
\hline Left & $3(100)$ & $0(0)$ & \\
\hline \multicolumn{4}{|l|}{ Tumor side, no. (\%) } \\
\hline Right & 0 & 0 & \\
\hline Left & 10 & 16 & \\
\hline Tumor location, no. (\%) & & & 0.78 \\
\hline Frontal & $4(50)$ & $4(50)$ & \\
\hline Temporal & $2(40)$ & $3(60)$ & \\
\hline Parietal & $3(50)$ & $3(50)$ & \\
\hline Insular & 0 & $1(100)$ & \\
\hline$>1$ lobe & $1(17)$ & $5(83)$ & \\
\hline Pathology, no. (\%) & & & 0.30 \\
\hline Grade II & $2(50)$ & $2(50)$ & \\
\hline Anaplastic & $2(40)$ & $3(60)$ & \\
\hline Glioblastoma & $6(35)$ & $11(65)$ & \\
\hline Previous operation, no. (\%) & & & 0.69 \\
\hline No & $6(35)$ & $11(65)$ & \\
\hline Yes & $4(44)$ & $5(56)$ & \\
\hline Preoperative deficits, no. (\%) & & & 0.25 \\
\hline No & $7(50)$ & $7(50)$ & \\
\hline Yes & $3(25)$ & $9(75)$ & \\
\hline DCS mapping, no. (\%) & & & 0.40 \\
\hline Negative & $7(47)$ & $8(53)$ & \\
\hline Positive & $2(25)$ & $6(75)$ & \\
\hline HFC resected, no. (\%) & & & 0.16 \\
\hline No & $4(67)$ & $2(33)$ & \\
\hline Yes & $6(30)$ & $14(70)$ & \\
\hline
\end{tabular}

$\mathrm{DCS}=$ direct cortical stimulation; $\mathrm{POD}=$ postoperative day.

told to describe "who is doing what to whom." For AN tests, patients were instructed to answer a question with a one-word response. Each task was scored based on the scoring guide as outlined in the QAB. ${ }^{1,42}$ Early postoperative language testing was performed on postoperative day 2 to 3 , and late postoperative testing was performed 1-3 months following the surgery. A decline in language task performance was defined by a change between baseline and early postoperative testing of at least 2.5 SDs from the mean.

\section{Statistical Analysis}

Descriptive statistics are reported as the mean $( \pm \mathrm{SD})$ for continuous variables and as the frequency of distribu- tion for categorical variables. The Pearson chi-square test was used for categorical analyses, and the Mann-Whitney test was performed for nonparametric comparisons of continuous variables. The Fisher exact test was used if more than $80 \%$ of values were less than 5 . Spearman correlation linear regressions compared the percentage of HFC resection with language task performance between baseline and postoperative scores. Statistics were analyzed using SPSS statistical software, version 24 (IBM Corp.) and Prism (GraphPad).

\section{Results \\ Subject Characteristics}

Demographic and clinical characteristics, with early postoperative language outcomes, are listed in Table 1. The study population included 10 women and 16 men, with a mean age of 50.8 years (range 20-80 years). Twenty-three of 26 patients (88.5\%) were right-handed. All patients had focal tumors located in the left hemisphere with language dominance confirmed by MEG. Eight patients (30.8\%) had tumors in the frontal lobe, $5(19.2 \%)$ had temporal lobe tumors, 6 (23.1\%) had parietal lobe tumors, 1 patient (3.8\%) had an insular tumor, and $6(23.1 \%)$ had tumors across multiple cortical regions. Nine patients $(34.6 \%)$ had undergone a prior operation (biopsy or resection), and 11 patients (42.3\%) had subjective self-reported baseline language dysfunction at the initial clinic visit. Early postoperative language assessment occurred an average of 2.3 days (range 1-8 days) after the operation.

\section{Surgical Characteristics}

All 26 patients underwent preoperative MEG for identification of resting-state functional connectivity network hubs (Fig. 2A). An average of $42.5 \mathrm{HFC}$ voxels (range 1-201) ipsilateral to the tumor were found in each patient. Twenty-three patients $(88.5 \%)$ proceeded with intraoperative speech mapping using an awake craniotomy. A total of 288 intraoperative sites were stimulated, with 11 sites (3.8\%) defined as positive for language errors (naming delays, paraphasic errors, and speech arrest). These intraoperative stimulation sites were not guided by preoperative MEG mapping. The mean percentage of HFC hubs resected within the ipsilateral hemisphere per patient was $11.8 \%$ (range $0 \%-50 \%$ ). Histopathological analysis confirmed low-grade glioma (WHO grade II) in 4 patients (15.4\%), anaplastic glioma (WHO grade III) in 5 patients $(19.2 \%)$, and glioblastoma (WHO grade IV) in 17 patients (65.3\%).

\section{Early Language Task Performance Following Resection of Intra- and Peritumoral HFC Hubs}

Between baseline and early postoperative testing, $\mathrm{QAB}$ language task performance declined by a mean difference of $-31.4 \%$ (range $-99 \%$ to $1.9 \%$; $=0.0023$, paired t-test). Across the multidimensional QAB assessment, our analysis focused on 5 domains specific to PN, TR, 4-SYL, $\mathrm{AN}$, and SYN language tasks, which were therefore used for outcome analysis. Overall performances in the QAB language task and across the 5 language tasks were not significantly different between baseline and intraoperative 

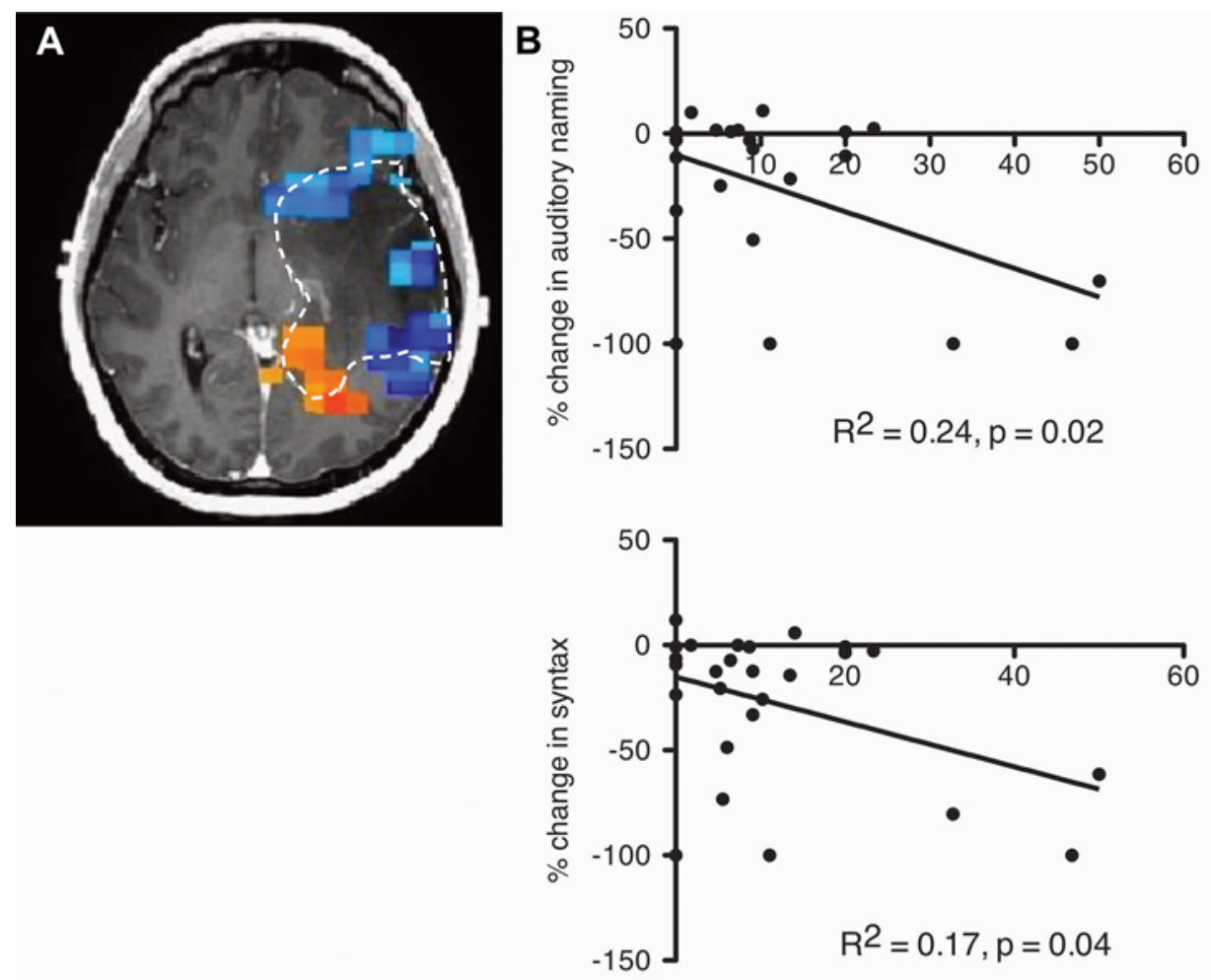

$\%$ of high functional connectivity hubs resected

FIG. 2. Resection HFC hubs correlates with worse outcomes in AN and SYN. A: Illustrative example of voxels with increased functional connectivity (red and orange) and suppressed functional connectivity (blue and green). Hashed line indicates the tumor outline. B: A patient's pre- and postoperative change in a specific language task (AN [upper]; SYN [lower]) was compared to the percentage of HFC hubs that were resected (AN: $R^{2}=0.24, p=0.02 ; S Y N: R^{2}=0.17, p=0.04$ ). Patients underwent preoperative MEG to identify resting-state HFC hubs, and resection was guided by intraoperative awake language mapping. Each dot represents a patient. Figure is available in color online only.

testing, with a mean difference of $-1.6 \%$ (SD 0.32) (range of means for the 5 language tasks: $-1.99 \%$ to $-1.41 \%$ ). Sixteen patients $(61.5 \%)$ exhibited early postoperative decline in at least one language task. Early postoperative language decline was most common on SYN $(54 \%, 14 / 26)$ and AN $(39 \%, 9 / 23)$ tests. Comparisons were made between patient sex, age, postoperative day of language evaluation, handedness, tumor location, pathology, history of previous surgery, baseline preoperative language deficits, positive IES mapping, and HFC sites resected in the cohorts with or without early postoperative language deficits (Table 1). Early language task decline was more common in righthanded patients than in left-handed individuals $(70 \%$ vs $0 \%, \mathrm{p}=0.04)$. No other comparisons reached statistical significance.

We next set out to determine whether the resection of IES-negative HFC network sites impacted early postoperative language task performance. We chose $25 \%$ as the threshold to stratify language outcomes because it bifurcated the range of HFC resected $(0 \%-50 \%)$ in our case series (Table 2). The mean overall QAB score decreased by $25.7 \%$ in patients with a $<25 \%$ HFC resection versus $60.2 \%$ with $\mathrm{a} \geq 25 \% \mathrm{HFC}$ resection $(\mathrm{p}=0.12)$. In the specific language tasks, removal of $\geq 25 \%$ of HFCs resulted in 3 of 3 patients having AN deficits compared to 6 of 20 patients $(100 \%$ vs $30 \%, \mathrm{p}=0.04)$ with $<25 \%$ HFCs resect- ed. No statistical significance was seen after stratification by the percentage of HFC resected for the other language tasks (TR, 4-SYL, PN, and SYN).

Since AN decline was found more commonly in patients after stratifying by percentage of HFC resected, we hypothesized that the number of HFC hubs resected would correlate with the severity of AN decline. Thus, we performed linear regression on the percentage change in postoperative AN scores to the percentage of HFC resected. We found that the resection of HFC hubs did indeed correlate with worse AN decline (Fig. 2B; $\mathrm{R}^{2}=0.24$, $\mathrm{p}$ $=0.02$ ). SYN testing also showed a significant negative correlation between performance and percentage of HFC resected (Fig. 2B; $\mathrm{R}^{2}=0.16, \mathrm{p}=0.04$ ). No significant correlations were found in the other language tasks.

We performed separate analyses to account for HFC voxels that might have been within new areas of FLAIR or diffusion weighted imaging (DWI) postoperatively. HFC voxels from two patients were identified within new areas of DWI signals. When excluding these patients from the linear regressions, or including the additional HFC voxels as resected voxels, the correlations remained significant between the degree of HFC loss and AN and SYN deficits (excluding patients, $\mathrm{p}=0.017$ and $\mathrm{p}=0.036$ for $\mathrm{AN}$ and SYN, respectively; including HFC voxels, $\mathrm{p}=0.022$ and $\mathrm{p}$ $=0.046$ for $\mathrm{AN}$ and $\mathrm{SYN}$, respectively). 
TABLE 2. Deficits in specific language tasks stratified by amount of HFC network hubs resected

\begin{tabular}{|c|c|c|c|}
\hline \multirow[b]{2}{*}{ Task } & \multicolumn{2}{|c|}{ Postop Deficit } & \multirow[b]{2}{*}{$p$ Value } \\
\hline & No & Yes & \\
\hline \multicolumn{4}{|l|}{ Text reading } \\
\hline HFC resected & & & 0.22 \\
\hline$<25 \%$ & $17(74)$ & $6(26)$ & \\
\hline$>25 \%$ & $1(33)$ & $2(67)$ & \\
\hline \multicolumn{4}{|c|}{ 4-syllable repetition } \\
\hline HFC resected & & & 0.17 \\
\hline$<25 \%$ & $18(78)$ & $5(22)$ & \\
\hline$>25 \%$ & $1(33)$ & $2(67)$ & \\
\hline \multicolumn{4}{|l|}{ Picture naming } \\
\hline HFC resected & & & 0.17 \\
\hline$<25 \%$ & $18(78)$ & $5(22)$ & \\
\hline$>25 \%$ & $1(33)$ & $2(67)$ & \\
\hline \multicolumn{4}{|l|}{ Syntax } \\
\hline HFC resected & & & 0.22 \\
\hline$<25 \%$ & $12(52)$ & $11(48)$ & \\
\hline$>25 \%$ & $0(0)$ & $3(100)$ & \\
\hline \multicolumn{4}{|l|}{ Auditory naming } \\
\hline HFC resected & & & 0.04 \\
\hline$<25 \%$ & $14(70)$ & $6(30)$ & \\
\hline$>25 \%$ & $0(0)$ & $3(100)$ & \\
\hline
\end{tabular}

Values are presented as the number $(\%)$ of patients.

\section{Late Language Task Performance Following Resection of Intra- and Peritumoral HFC Hubs}

Next, we wanted to understand whether the effects of HFC resection and AN and SYN performance extended beyond the early postoperative period. We therefore performed a second language assessment following glioma surgery. Sixty-nine percent of patients (18/26) with an average follow-up of 2.2 months (range 1-6 months) completed additional testing for late language task performance. At this later postoperative language assessment time point, there was no longer a correlation between HFC resection and $\mathrm{AN}$ and SYN task performance $\left(\mathrm{AN}: \mathrm{R}^{2}=0.00, \mathrm{p}=\right.$ 0.94; SYN: $\mathrm{R}^{2}=0.00, \mathrm{p}=1.00$ [Fig. 3]). In the 11 patients with initial AN and SYN decline detected at early postoperative testing, all recovered to their preoperative baseline status with the exception of two individuals (Fig. 4). For patient 1 , a tailored resection was performed and allowed identification of two IES-positive cortical language sites during awake language mapping, resulting in the removal of $13.5 \%$ of HFC hubs (Fig. 4A-E). At late postoperative testing, both AN and SYN remained impaired (AN: $-23 \%$, SYN: $-13 \%$ ), while all other language tasks remained unchanged from preoperative baseline (PN: $-12 \%$; TR: 0\%; 4-SYL: $-1 \%$ ). Postoperative diffusion tensor imaging/ MRI in patient 1 revealed a focal region of restricted diffusion involving the left inferior frontooccipital fasciculus (Fig. 4D-F). For patient 2, a tailored resection resulted in loss of $9 \%$ of HFC sites (Fig. 4G-I). At late follow-up, the scores in all 5 language tasks declined significantly from preoperative baseline assessments (PN: $-100 \%$; TR:
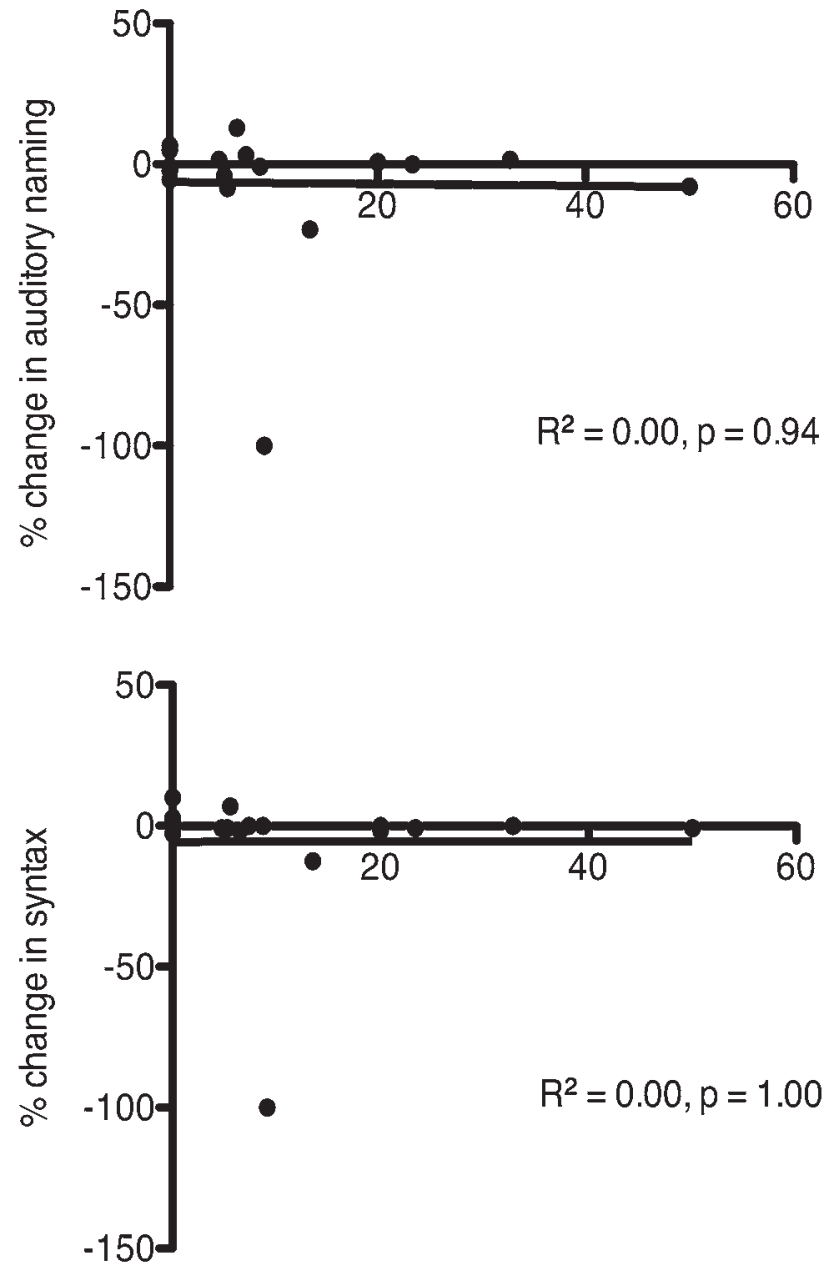

\section{$\%$ of high functional connectivity hubs resected}

FIG. 3. Degree of HFC resection is not correlated with language task performance at later follow-up. Changes in AN (upper) and SYN (lower) language tasks were not correlated to the percentage of $\mathrm{HFC}$ resected at later follow-up (AN: $\left.R^{2}=0.00, p=0.94 ; S Y N: R^{2}=0.00, p=1.00\right)$.

$-16 \%$; 4-SYL: $-21 \%$; AN: $-100 \%$, SYN: $-100 \%)$. Subsequent MRI demonstrated new contrast enhancement consistent with tumor progression (Fig. 4G-I). The remaining 9 patients with early postoperative language deficits recovered to their preoperative baselines.

\section{Illustrative Case}

A 41-year-old right-handed man with a left frontaltemporal anaplastic oligodendroglioma presented to our service after a generalized seizure. Imaging revealed a minimally enhancing infiltrative lesion within the anterior frontal-temporal lobes (Fig. 5A). The patient had no subjective complaints of aphasia at presentation. Preoperative MEG of resting-state functional connectivity revealed 4 HFC sites within the left hemisphere. Three HFC sites were located within the tumor (Fig. 5B; a white dotted line demarcates tumor margin surrounding an HFC hub in that section). Baseline preoperative language assessment was 
Patient \#1:
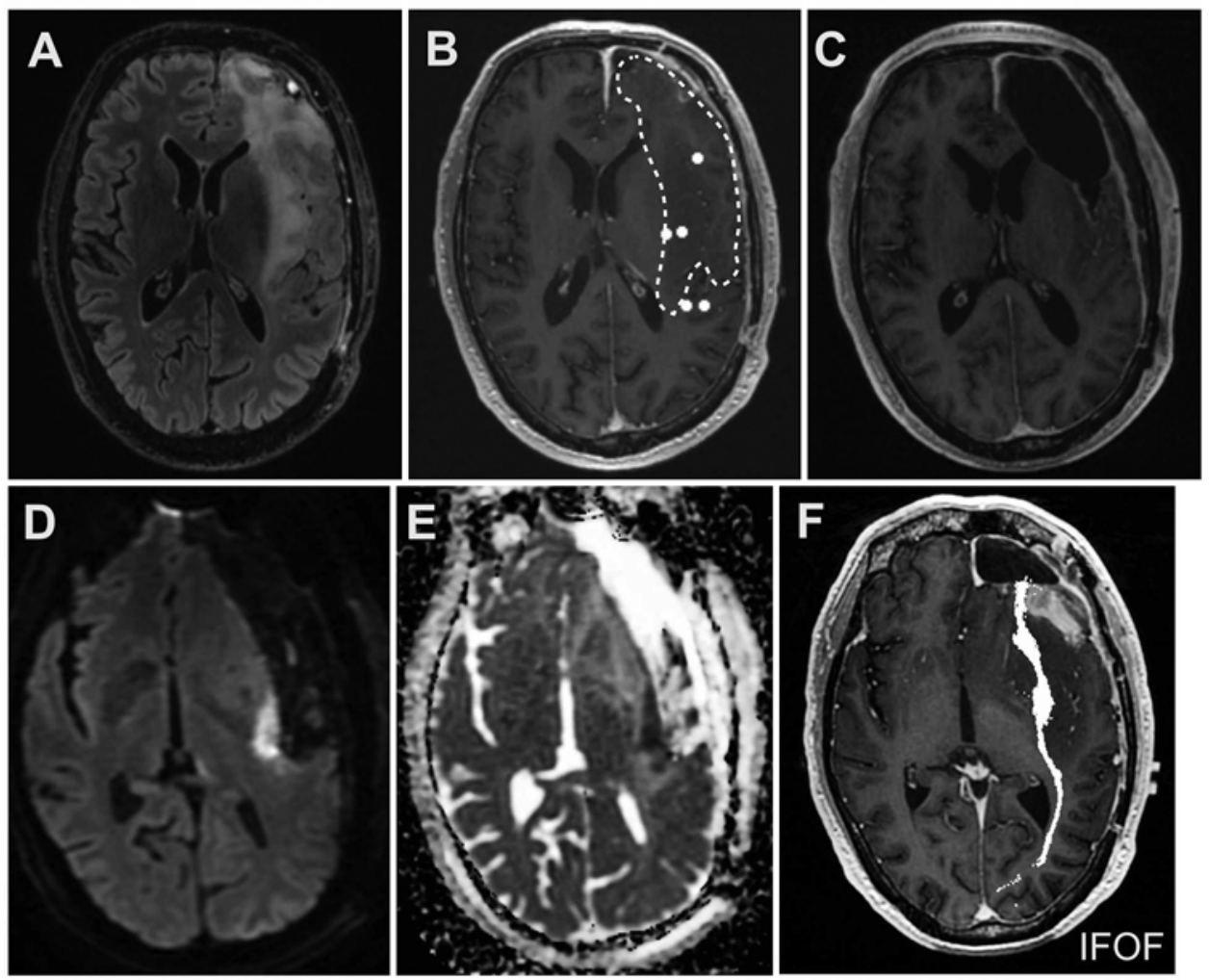

\section{Patient \#2:}
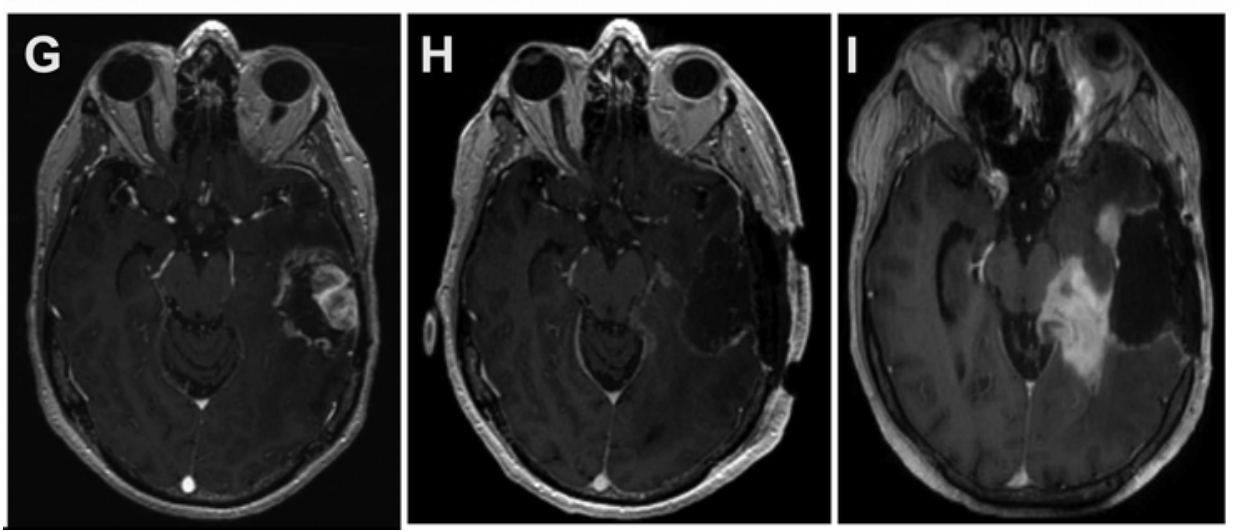

FIG. 4. Patients in whom $A N$ and SYN function had failed to recover by their late postoperative follow-up visit. Upper: Patient 1 had not recovered to baseline AN and SYN task performance at late follow-up. A: Preoperative T2-weighted FLAIR MR image showing a frontal-temporal-insular tumor. B: Resting-state MEG image overlaid onto T1-weighted Gd-enhanced MR image with white dots representing HFC nodes. Hashed lines denote tumor margin. C: Postoperative T1-weighted Gd-enhanced MR image showing the resection cavity. D-F: Postoperative DWI and ADC (apparent diffusion coefficient) images revealing a focal area of stroke directly in the inferior frontooccipital fasciculus (IFOF). Lower: Patient 2 had worsened performance in all language tasks at late follow-up. G: Preoperative T1-weighted Gd-enhanced MR image showing a temporal tumor. H: Postoperative image of the resection cavity. I: One-month follow-up image showing new contrast enhancement consistent with tumor progression.

performed, including the $\mathrm{QAB}$ and the 5 language tasks (Fig. 1). The patient then underwent awake IES language mapping using standard clinical protocol. IES of 3 language sites (sites 20-22) resulted in anomia and they were therefore preserved, and a $95 \%$ extent of resection was achieved (Fig. 5C and D). Fifty percent of the HFC hubs $(2 / 4)$ were resected during tumor removal. Compared to preoperative assessments, early postoperative language evaluation on postoperative day 2 illustrated a decline in overall QAB by $21 \%$. The patient experienced a decline in SYN (-61\% compared to baseline) and AN (-70\% compared to baseline) in the early postoperative period. At late follow-up testing, the patient's SYN and AN results had recovered to preoperative baseline. 

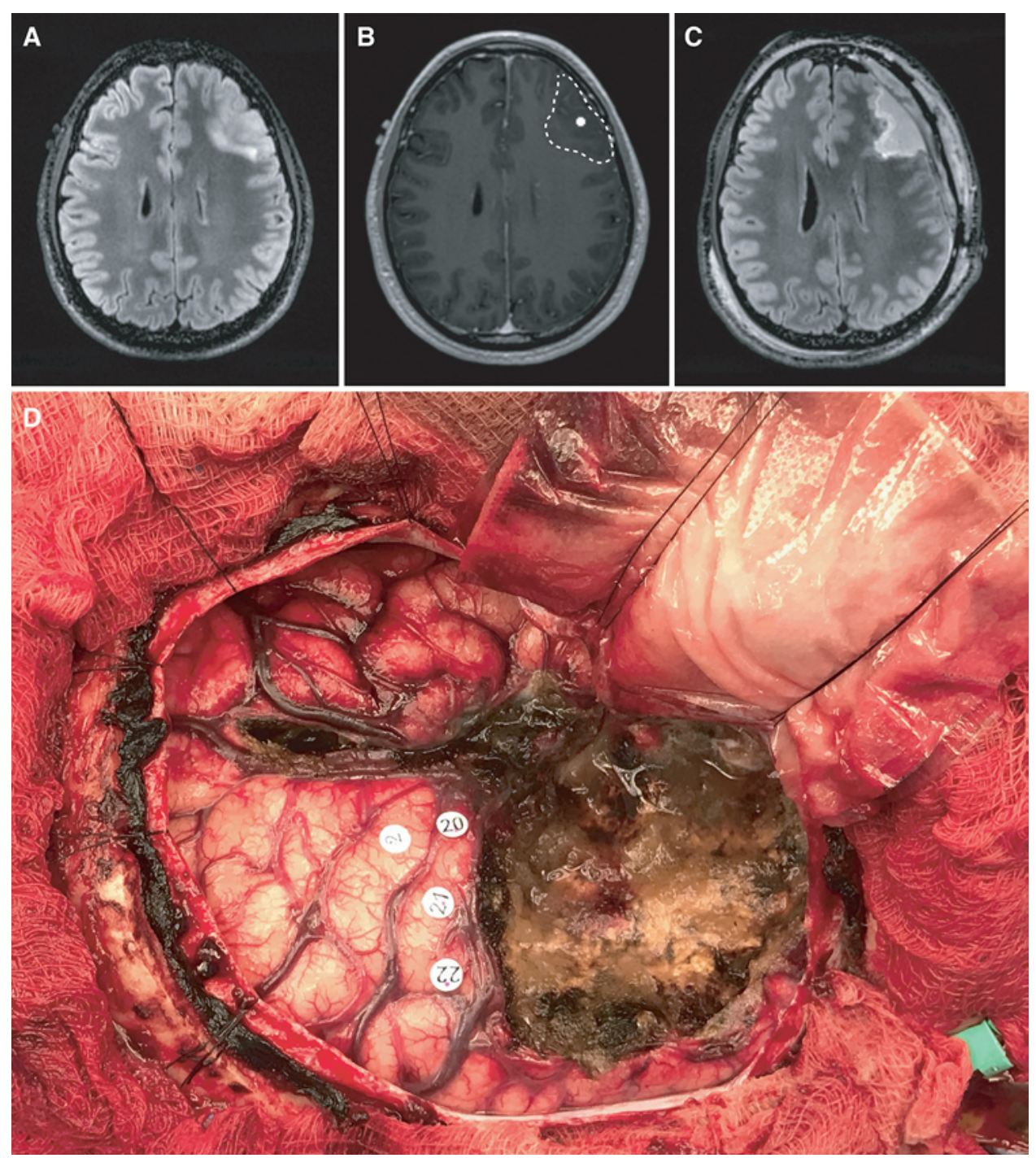

FIG. 5. An illustrative case. A 41-year-old right-handed man with a left frontal-temporal anaplastic oligodendroglioma status after he underwent a debulking procedure at an outside hospital. The patient had no preoperative language deficits. A: Preoperative T2-weighted FLAIR MR image revealing an infiltrative T2 hyperintensity within the left anterior frontal lobe. B: Preoperative MEG image of an HFC hub superimposed on the preoperative T1-weighted post-Gd MR image. The tumor margin is demarcated by the hashed line. C: Postoperative T2-weighted FLAIR image showing a 95\% extent of resection. D: Intraoperative photograph taken after tumor resection. Number 2 represents the face motor cortex. Numbers 20, 21, and 22 mark anomia sites identified by IES. Figure is available in color online only.

\section{Discussion}

Preservation of language is a critical component in the surgical management of patients with dominant-hemisphere low- and high-grade gliomas. Maximizing the extent of tumor resection in a focal manner is thought to have broader implications on short- and long-range neural networks. Standard surgical technique applies IES of cortical and subcortical foci, but it remains unknown why electrical stimulation along these seemingly critical points of the broader network results in behavioral or cognitive changes, while stimulation along adjacent areas of the network remains behaviorally silent. Removal of IES-positive tumoral and peritumoral sites during surgery is not within standard clinical practice. However, it remains unknown whether the removal of IES-negative HFC sites impacts cognitive or language processing. A more complete understanding of how HFC networks contribute to language processing is important for both surgical planning and patient counseling. This study suggests that the percentage of IES-negative HFC sites removed during surgery correlates with early postoperative AN and SYN language task performance. However, with the exception of postoperative stroke or tumor progression, task performance recovers to preoperative baseline in all patients.

\section{HFC Network Hubs and Early Postoperative Language Performance}

We have previously shown that functional connectivity analyses, as determined by imaginary coherence from MEG, are a promising tool for predicting postsurgical 
neurological outcomes. LFC regions, in particular, have a $100 \%$ positive predictive value for negative IES,,$^{20}$ and resection of LFC network sites resulted in $29 \%$ and $0 \%$ rates of new neurological deficit at 1-week and 6-month follow-up visits, respectively. ${ }^{36}$ In contrast, our understanding of HFC regions is more ambiguous. With a positive predictive value for positive IES of only $64 \%,,^{20}$ how should preoperative identification of HFC sites influence surgical planning? While IES-positive HFC sites may overlap, intraoperative decisions are made based on IES, not functional connectivity measures. Therefore, we studied the short- and long-term effects of resecting HFCpositive, IES-negative sites. Indeed, an average of $11.8 \%$ of total intra- and peritumoral HFC sites were resected in this series, and removal of HFC sites resulted in an early postoperative language decline in $70 \%$ of patients $(14 / 20)$. We found a significant negative correlation between the percentage of HFC resected and early language outcomes in AN and SYN. This result suggests that HFC-positive, IES-negative sites may indeed contribute to network-level language processing.

Our rate of early postoperative language decline (mean postoperative day 2.3) is within the range of $23 \%-100 \%$ reported in recent studies of tumor resection and IES. $5,11,30,43$ Prior published reports illustrate that following a transient decline, aphasia resolves in the majority of patients. Potential mechanisms explaining development of new transient language deficits include postsurgical edema, postsurgical altered cortical neuron metabolism, and loss of functional IES-negative sites within and surrounding the resection area. ${ }^{43}$

\section{Differences in Studies Examining Postoperative Language Outcomes and IES}

An important distinction between studies examining postoperative language outcomes of IES and our current series lies in the graded relative complexity of language assessments. Traditionally, speech and language evaluations during IES consist of counting, PN, TR, and sentence completion. ${ }^{11,24,29,30}$ Outside of the operating room setting, a broader range of tasks may be employed, thereby examining more complex relationships. During AN, for example, participants are asked to answer questions with a one-word response following a nonvisual, auditory stimulus (e.g., "What is a fruit that makes wine?" "Grape"). These questions differ from PN tasks traditionally used in IES, in that they contain a receptive component requiring the participant to first process the semantic meaning of the question stem without a visual stimulus. Based on the "dual streams" model of language organization, processing of AN and SYN tasks may activate a wider range of cortical brain regions via dorsal and ventral streams (e.g., superior longitudinal fasciculus and inferior frontooccipital fasciculus, respectively). ${ }^{8}{ }^{816}$ Recent fMRI studies have further demonstrated that incorporating increasingly complex tasks, such as grammar tests or SYN, uncovers a larger volume of BOLD (blood oxygen level-dependent) signal activation throughout the dominant hemisphere. ${ }^{25,40}$

It remains unclear why IES methods do not uncover all HFC network sites. It is believed that IES-positive sites have greater functional connectivity to surrounding cor- tex compared with IES-negative sites. ${ }^{27}$ IES relies on language tasks that are rapid and highly reliable; therefore, $\mathrm{PN}$ remains the workhorse of intraoperative testing. It is therefore possible that PN and TR IES language mapping may not require long-range network recruitment of HFC-dependent networks. In this study, correlations between the percentage of HFC sites resected and language decline are seen only in AN and SYN tasks. Perhaps AN and SYN necessitate wider language network contributions and are more representative of, or are reliant on, HFC sites. The specific roles of HFC network hubs in language processing are poorly understood. One difficulty in studying these important relationships is the ongoing plasticity of the adult brain, particularly with respect to language in the everchanging landscape of adult glioma. In this patient population, the mechanisms of aphasia recovery are likely impacted by patient-specific factors, tumor biology, and oncological therapies such as brain irradiation.

In this study, we intentionally highlighted our language outcomes for both the early postoperative period (mean 2.3 days) and the late postoperative period (mean 2.2 months) following surgical intervention. ${ }^{11,24,29,30}$ By analyzing language outcomes within this focused time period, the direct effects of HFC site removal could be captured by minimizing additional oncological and therapeutic variables, all of which are thought to impact CNS neuroplasticity. ${ }^{11,26,}$ ${ }_{30,43}$ Indeed, even with our focused perioperative approach, one patient in this study failed to recover from initial AN and SYN deficits due to early tumor progression within 1 month of surgery (Fig. 4G-I).

\section{Complementary Language Mapping and HFC Tissue as a Compensatory Biomarker}

Although direct IES with awake language mapping remains the gold standard for minimizing the resection of functional tissue, our study supports the use of complementary preoperative MEG functional connectivity to anticipate the risk of early postoperative language decline. Counseling patients about early and late neurological impairments facilitates informed medical decision-making. Consistent with a prior study, ${ }^{36}$ negative IES stimulation alone could not predict early language outcomes for all tasks employed in our cohort (Table 1). In the long term, however, AN and SYN language responses recovered to baseline regardless of the HFC sites resected across patients. The mechanisms of aphasia recovery are poorly understood; however, they are likely influenced by processes such as glioma-neuronal interactions, activation of latent functional pathways, and secreted proteins influenced by gene polymorphisms. ${ }^{2}$ Recently, Traut et al. used wholebrain MEG activity in an auditory verb-generation task to demonstrate functional plasticity, including increased lateralization of language activation following resection. ${ }^{39}$ In order to better understand how network interactions for language processing occur across a variety of language domains, future studies will incorporate AN and SYN language tasks during IES. Finally, in our MEG analyses, HFC sites are operationally defined as ipsilateral to the side of the tumor, raising the interesting possibility that HFC sites themselves represent compensatory regions for language processing (i.e., cells within these regions may 
contain active or latent neuron-neuron or neuroglial interactions supporting network connectivity). ${ }^{10,11}$

\section{Limitations}

The small sample size in our study was not sufficiently powered to uncover significant differences in multiple univariate comparisons and precluded multivariate analyses. Conclusions from our study should be tempered until verification with larger sample sizes. Additionally, imaginary coherence measures of functional connectivity were performed only at the perioperative baseline time period. Ideally, HFC network connectivity data would be available at each time point; however, longitudinal MEG at baseline, 2 days, and 2-3 months following surgery was not feasible for this study. Because patients received a standardized decadron course and taper off in 14 days, we find it unlikely to have confounded the findings in our current study.

\section{Conclusions}

Surgical removal of IES-negative HFC sites correlated with early decline in AN and SYN language task performance. Task performance recovered to preoperative baseline at later time points in all patients who did not experience tumor progression or a postoperative stroke. These data suggest that functional connectivity measured by MEG may serve as a useful imaging predictor of early decline in language processing.

\section{Acknowledgments}

Funding was received from the following: NIH NINDS (K08 12649025), Robert Wood Johnson Foundation (74259), NIH NCI SPORE (5P50CA097257-15), and the LoGlio Collective.

\section{References}

1. Aabedi AA, Ahn E, Kakaizada S, Valdivia C, Young JS, Hervey-Jumper $\mathrm{H}$, et al: Assessment of wakefulness during awake craniotomy to predict intraoperative language performance. J Neurosurg [epub ahead of print May 31, 2019. DOI: 10.3171/2019.2.JNS183486]

2. Altshuler DB, Wang L, Zhao L, Miklja Z, Linzey J, Brezzell A, et al: $B D N F, C O M T$, and $D R D 2$ polymorphisms and ability to return to work in adult patients with low- and highgrade glioma. Neurooncol Pract 6:375-385, 2019

3. Bartolomei F, Bosma I, Klein M, Baayen JC, Reijneveld JC, Postma TJ, et al: Disturbed functional connectivity in brain tumour patients: evaluation by graph analysis of synchronization matrices. Clin Neurophysiol 117:2039-2049, 2006

4. Bartolomei F, Bosma I, Klein M, Baayen JC, Reijneveld JC, Postma TJ, et al: How do brain tumors alter functional connectivity? A magnetoencephalography study. Ann Neurol 59:128-138, 2006

5. Benzagmout M, Gatignol P, Duffau H: Resection of World Health Organization Grade II gliomas involving Broca's area: methodological and functional considerations. Neurosurgery 61:741-753, 2007

6. Bosma I, Douw L, Bartolomei F, Heimans JJ, van Dijk BW, Postma TJ, et al: Synchronized brain activity and neurocognitive function in patients with low-grade glioma: a magnetoencephalography study. Neuro Oncol 10:734-744, 2008

7. Bosma I, Stam CJ, Douw L, Bartolomei F, Heimans JJ, van Dijk BW, et al: The influence of low-grade glioma on resting state oscillatory brain activity: a magnetoencephalography study. J Neurooncol 88:77-85, 2008
8. Chang EF, Raygor KP, Berger MS: Contemporary model of language organization: an overview for neurosurgeons. J Neurosurg 122:250-261, 2015

9. Dalal SS, Guggisberg AG, Edwards E, Sekihara K, Findlay AM, Canolty RT, et al: Five-dimensional neuroimaging: localization of the time-frequency dynamics of cortical activity. Neuroimage 40:1686-1700, 2008

10. Duffau H: New concepts in surgery of WHO grade II gliomas: functional brain mapping, connectionism and plasticity-a review. J Neurooncol 79:77-115, 2006

11. Duffau H, Capelle L, Denvil D, Sichez N, Gatignol P, Lopes $\mathrm{M}$, et al: Functional recovery after surgical resection of low grade gliomas in eloquent brain: hypothesis of brain compensation. J Neurol Neurosurg Psychiatry 74:901-907, 2003

12. Guggisberg AG, Honma SM, Findlay AM, Dalal SS, Kirsch $\mathrm{HE}$, Berger MS, et al: Mapping functional connectivity in patients with brain lesions. Ann Neurol 63:193-203, 2008

13. Hari R, Levänen S, Raij T: Timing of human cortical functions during cognition: role of MEG. Trends Cogn Sci 4:455-462, 2000

14. Hervey-Jumper SL, Berger MS: Role of surgical resection in low- and high-grade gliomas. Curr Treat Options Neurol 16:284, 2014

15. Hervey-Jumper SL, Li J, Lau D, Molinaro AM, Perry DW, Meng L, et al: Awake craniotomy to maximize glioma resection: methods and technical nuances over a 27 -year period. J Neurosurg 123:325-339, 2015

16. Hickok G, Poeppel D: The cortical organization of speech processing. Nat Rev Neurosci 8:393-402, 2007

17. Hinkley LB, Owen JP, Fisher M, Findlay AM, Vinogradov S, Nagarajan SS: Cognitive impairments in schizophrenia as assessed through activation and connectivity measures of magnetoencephalography (MEG) data. Front Hum Neurosci 3:73, 2010

18. Keles GE, Lundin DA, Lamborn KR, Chang EF, Ojemann G, Berger MS: Intraoperative subcortical stimulation mapping for hemispherical perirolandic gliomas located within or adjacent to the descending motor pathways: evaluation of morbidity and assessment of functional outcome in 294 patients. J Neurosurg 100:369-375, 2004

19. Lin PT, Berger MS, Nagarajan SS: Motor field sensitivity for preoperative localization of motor cortex. J Neurosurg 105:588-594, 2006

20. Martino J, Honma SM, Findlay AM, Guggisberg AG, Owen JP, Kirsch HE, et al: Resting functional connectivity in patients with brain tumors in eloquent areas. Ann Neurol 69:521-532, 2011

21. Nagarajan S, Kirsch H, Lin P, Findlay A, Honma S, Berger MS: Preoperative localization of hand motor cortex by adaptive spatial filtering of magnetoencephalography data. J Neurosurg 109:228-237, 2008

22. Nolte G, Bai O, Wheaton L, Mari Z, Vorbach S, Hallett M: Identifying true brain interaction from EEG data using the imaginary part of coherency. Clin Neurophysiol 115:22922307, 2004

23. Ojemann G, Ojemann J, Lettich E, Berger M: Cortical language localization in left, dominant hemisphere. An electrical stimulation mapping investigation in 117 patients. J Neurosurg 71:316-326, 1989

24. Ojemann JG, Miller JW, Silbergeld DL: Preserved function in brain invaded by tumor. Neurosurgery 39:253-259, 1996

25. Połczyńska M, Japardi K, Curtiss S, Moody T, Benjamin C, Cho A, et al: Improving language mapping in clinical fMRI through assessment of grammar. Neuroimage Clin 15:415427, 2017

26. Racine CA, Li J, Molinaro AM, Butowski N, Berger MS: Neurocognitive function in newly diagnosed low-grade glioma patients undergoing surgical resection with awake mapping techniques. Neurosurgery 77:371-379, 2015 
27. Rolston JD, Chang EF: Critical language areas show increased functional connectivity in human cortex. Cereb Cortex 28:4161-4168, 2018

28. Sanai N, Berger MS: Glioma extent of resection and its impact on patient outcome. Neurosurgery 62:753-764, 264266, 2008

29. Sanai N, Berger MS: Intraoperative stimulation techniques for functional pathway preservation and glioma resection. Neurosurg Focus 28(2):E1, 2010

30. Sanai N, Mirzadeh Z, Berger MS: Functional outcome after language mapping for glioma resection. N Engl J Med 358:18-27, 2008

31. Sanai N, Polley MY, McDermott MW, Parsa AT, Berger MS: An extent of resection threshold for newly diagnosed glioblastomas. J Neurosurg 115:3-8, 2011

32. Sartorius CJ, Berger MS: Rapid termination of intraoperative stimulation-evoked seizures with application of cold Ringer's lactate to the cortex. Technical note. J Neurosurg 88:349351,1998

33. Schiffbauer H, Berger MS, Ferrari P, Freudenstein D, Rowley HA, Roberts TP: Preoperative magnetic source imaging for brain tumor surgery: a quantitative comparison with intraoperative sensory and motor mapping. Neurosurg Focus 15(1):E7, 2003

34. Smith JS, Chang EF, Lamborn KR, Chang SM, Prados MD, Cha S, et al: Role of extent of resection in the long-term outcome of low-grade hemispheric gliomas. J Clin Oncol 26:1338-1345, 2008

35. Southwell DG, Hervey-Jumper SL, Perry DW, Berger MS: Intraoperative mapping during repeat awake craniotomy reveals the functional plasticity of adult cortex. J Neurosurg 124:1460-1469, 2016

36. Tarapore PE, Martino J, Guggisberg AG, Owen J, Honma SM, Findlay A, et al: Magnetoencephalographic imaging of resting-state functional connectivity predicts postsurgical neurological outcome in brain gliomas. Neurosurgery 71:1012-1022, 2012

37. Tarapore PE, Tate MC, Findlay AM, Honma SM, Mizuiri D, Berger MS, et al: Preoperative multimodal motor mapping: a comparison of magnetoencephalography imaging, navigated transcranial magnetic stimulation, and direct cortical stimulation. J Neurosurg 117:354-362, 2012

38. Thiel A, Herholz K, Koyuncu A, Ghaemi M, Kracht LW, Habedank B, et al: Plasticity of language networks in patients with brain tumors: a positron emission tomography activation study. Ann Neurol 50:620-629, 2001

39. Traut T, Sardesh N, Bulubas L, Findlay A, Honma SM, Mizuiri D, et al: MEG imaging of recurrent gliomas reveals functional plasticity of hemispheric language specialization. Hum Brain Mapp 40:1082-1092, 2019

40. Unadkat P, Fumagalli L, Rigolo L, Vangel MG, Young GS, Huang R, et al: Functional MRI task comparison for language mapping in neurosurgical patients. J Neuroimaging 29:348-356, 2019

41. Vrba J, Robinson SE: Signal processing in magnetoencephalography. Methods 25:249-271, 2001

42. Wilson SM, Eriksson DK, Schneck SM, Lucanie JM: A quick aphasia battery for efficient, reliable, and multidimensional assessment of language function. PLoS One 13:e0192773-e29, 2018

43. Wilson SM, Lam D, Babiak MC, Perry DW, Shih T, Hess CP, et al: Transient aphasias after left hemisphere resective surgery. J Neurosurg 123:581-593, 2015

44. Wipf D, Nagarajan S: A unified Bayesian framework for MEG/EEG source imaging. Neuroimage 44:947-966, 2009

45. Yang TT, Gallen CC, Schwartz BJ, Bloom FE: Noninvasive somatosensory homunculus mapping in humans by using a large-array biomagnetometer. Proc Natl Acad Sci U S A 90:3098-3102, 1993

\section{Disclosures}

The authors report no conflict of interest concerning the materials or methods used in this study or the findings specified in this paper.

\section{Author Contributions}

Conception and design: Hervey-Jumper, Lee, Berger. Acquisition of data: Hervey-Jumper, Lee, Kakaizada, Valdivia, Findlay, Berger. Analysis and interpretation of data: Hervey-Jumper, Lee, Faltermeier. Drafting the article: Hervey-Jumper, Lee. Critically revising the article: Hervey-Jumper, Lee, Morshed, Young, Findlay, Nagarajan, Berger. Reviewed submitted version of manuscript: all authors. Approved the final version of the manuscript on behalf of all authors: Hervey-Jumper. Statistical analysis: Lee, Faltermeier. Administrative/technical/material support: Hervey-Jumper, Kakaizada, Valdivia, Findlay, Nagarajan, Berger. Study supervision: Hervey-Jumper, Berger.

\section{Correspondence}

Shawn L. Hervey-Jumper: University of California, San Francisco, CA. shawn.hervey-jumper@ucsf.edu. 